\title{
Un equipo en juego, con toda la mar detrás. Procesos de trabajo y producción de Salud Mental ${ }^{1}$
}

\section{A team at stake, with the whole sea behind them. Work Processes and Production of Mental Health}

\author{
Elisa Bellezze ${ }^{2}$
}

\section{Resumen}

El artículo aborda los procesos de trabajo del equipo interdisciplinario e interinstitucional de Puente de Tierra, una experiencia territorial de juego con niños, niñas y jóvenes en contextos de pobreza de la ciudad de Rosario. Se describe el dispositivo lúdico y su inscripción/tensión respecto a la política pública. Luego, se reconstruye el proceso de trabajo durante el período estudiado, ordenando la información en torno a cuatro aspectos relevantes. Además, se articulan conceptualizaciones que dimensionan, por un lado, las dificultades de la práctica, y por otro, los recursos y herramientas clínicas con las que se cuenta para afrontarlas. El equipo en juego implica un entramado colectivo vinculado con la producción de salud mental, donde es central la dimensión per-elaborativa, creativa y de pensamiento crítico. Finalmente se esbozan reflexiones y nuevos interrogantes.
Palabras clave: Experiencia - Trabajo - Juego

- Equipo - Salud mental - Crueldad - Ternura

\section{Summary}

The article discusses the working processes of the interdisciplinary and the inter-institutional team Puente de Tierra, a territorial experience of play with children and young people in contexts of poverty in the city of Rosario. It describes the playful device and its registration/tension with respect to public policy. Then, the work process is reconstructed during the period studied, ordering the information around four relevant aspects. In addition, conceptualizations are articulated: on the one hand, the difficulties of the practice, and on the other, the clinical resources and tools that are available to face them. The team at stake involves a collective network linked to the production of mental health, where the

\footnotetext{
${ }^{1}$ El presente artículo fue escrito sobre la base del Trabajo Final Integrador (TIF) de la Carrera de Especialización en Psicología Clínica Institucional y Comunitaria (UNR). "Con toda la mar detrás. Procesos de trabajo de un equipo interdisciplinario e interinstitucional y experiencia territorial de juego con niños, niñas y jóvenes en contextos de pobreza" del que soy autora y que fue dirigido por Lic. Marta Basile, directora de la experiencia Barriletes en Bandada (Neuquén, Argentina).

${ }^{2}$ Psicóloga, Profesora de Psicología, Especialista en Psicología Clínica, Institucional y Comunitaria. UNR. Trabaja en consultorio particular y en el ámbito público. Actualmente se desempeña como Psicóloga en el centro de salud provincial n³, 7 de septiembre, y como Acompañante Terapéutica de la Dirección de Salud Mental de la Provincia de Santa Fe. Desde su paso por la Universidad es militante del proyecto colectivo Vamos Patria Grande. Feminista y activista por los derechos humanos, de las infancias y juventudes y de los/as trabajadores/ as. elisabellezze@yahoo.com.ar
} 
per-elaborative, creative and critical-thinking dimension is central. Finally, reflections and new questions are outlined.

Keywords: Experience - Work-Team - Game - Mental Health - Cruelty - Tenderness

\section{Introducción}

Reflexionar sobre la práctica, sistematizar experiencias y teorizar lo hecho, son tareas que no siempre logran tomar cuerpo en el devenir cotidiano que transcurre en instituciones públicas. Valorizar los relatos, registrarlos y ponerlos a circular es uno de los motivos de esta escritura.

Este artículo plasma un recorrido que analiza los procesos de trabajo de un equipo interdisciplinario e interinstitucional en una experiencia territorial de juego con niños, niñas y jóvenes en contextos de pobreza. ${ }^{3} \mathrm{El}$ dispositivo lúdico Puente de Tierra comienza en el año 2009 y tiene un desarrollo de diez años, hasta su cese en 2018.

A los fines prácticos de la conceptualización se hace uso de una abstracción para referir al equipo en tercera persona; sin embargo, la autora se constituye como actor de la experiencia, por haber participado en dicho equipo durante la Residencia de Posgrado realizada desde octubre 2012 a diciembre 2014 en un centro de salud de la ciudad de Rosario. El estudio llevado a cabo se circunscribe a dicho período.

Aquí se refleja sintéticamente dicho estudio que instala la propia mirada hacia el equipo del que se formó parte. Ese mirar lo que hacemos, lo que somos, y el cómo nos soste-

\footnotetext{
${ }^{3}$ Se omite intencionalmente el nombre del dispositivo donde se desempeña el equipo estudiado, y se lo nombra en adelante "Puente de Tierra", por razones de reserva y confidencialidad de las personas involucradas.
}

nemos y sostenemos el trabajo en contextos de dificultad supone un movimiento reflexivo propio de la producción de pensamiento crítico. Así, se explicita la lógica del proceso vivido y se estudian los factores intervinientes en dicho proceso. La sistematización de experiencias, según Jara Holliday (2012), es un ejercicio de producción de conocimiento crítico vinculado a los dilemas de una práctica social y a los saberes que ella produce y tiene el propósito general de conocer la realidad para transformarla. Pensarnos como sujetos de las prácticas no solo da sentido a las mismas, sino también nos resguarda del arrasamiento (de las consecuencias trágicas) que produce la crueldad de los territorios que habitamos.

El trabajo en instituciones públicas que desarrollan sus prácticas en contexto de pobreza y territorios signados por la violencia y el desamparo, confronta al equipo que se estudia con el gran dilema de asistir al escándalo de la miseria, en palabras de Ulloa (1995). Y, sin embargo, Jugar junto a niños, niñas y jóvenes en estas condiciones, y a pesar de ellas, es el desafío cotidiano, y el saber que supone Puente de Tierra.

Subrayar la vigencia de los interrogantes planteados en ese estudio implica no solo dimensionar las dificultades que enfrentan los equipos de trabajo en el ámbito público, abocados a una práctica a la intemperie; sino también valorar los recursos, estrategias, modos y herramientas clínicas con que se cuenta para afrontarlas. Por lo mismo, el foco del desarrollo se coloca sobre el proceso de trabajo del equipo que lleva a delante la experiencia. Algunas de las preguntas que inician el desarrollo del escrito son: ¿Qué es Puente de Tierra? ¿Dónde trabaja este equipo? ¿Cómo se conforma? ¿Cómo se organizan las tareas? ¿Qué aspectos el equipo valora positivamente? ¿Qué dificultades se encuentran? ¿Cuál es 
la respuesta frente a las mismas?

En primer lugar, se describen el dispositivo Puente de Tierra y el territorio donde se despliega, y se sitúa su inscripción/tensión respecto a la política pública. A continuación, se reconstruye el proceso de trabajo del equipo durante el período estudiado, con la ayuda de una guía que ordena la información en torno a cuatro aspectos importantes. Luego, se articulan conceptualizaciones en torno al mundo del trabajo y su gestión-organización, a las funciones del equipo en tanto adultos/as en juego (actividades recreativas) con niños/ as y jóvenes, y a lo que se da a llamar producción de salud mental. Por último, se esbozan algunas reflexiones.

\section{Puente de tierra. (características y ten- siones)}

El dispositivo de juego Puente de tierra tiene como característica fundamental ser una experiencia territorial. En su denominación, y en las prácticas que despliega, se sostiene una pregunta en relación a los lazos (los puentes) $y$ al territorio (la tierra). El modo en que el equipo responde a esto constituye su singularidad. El trabajo con la historia, los mitos, la vida y la identidad barrial hacen un camino, un puente, hacia la construcción de una identidad común.

El marco de esta propuesta nacida en el año dos mil nueve se delinea en un documento inédito, de elaboración colectiva por parte del equipo de trabajo:

El proyecto "Puente de tierra" se propone como un espacio semanal de encuentro para el juego con niños, niñas y jóvenes entre 10 y 14 años. Se trata de una experiencia interdisciplinaria (psicólogos, trabajadores sociales, médi- cos, enfermeros, profesores de educación física, artistas, referentes barriales, estudiantes), intersectorial (área salud, promoción social, cultura, extensión universitaria), y en situación, con el eje puesto en la producción de subjetividad y en la construcción de lo público como posible espacio habitable para todos/as.

Apunta a promover el habitar en el espacio público considerando las condiciones propias de un territorio donde prima la ruptura del lazo social. Se trata de fortalecer el encuentro entre y con las instituciones público estatal y el acontecer del barrio, construir puentes que nos acerquen, formular relatos que interroguen y desnaturalicen. En este sentido hablamos de nuevas institucionalidades, ya que nos proponemos promover un relato historizado, vivo, concreto del territorio que trascienda el caso por caso, o la lectura de los problemas ya conocidos como problemas, o el etiquetamiento de los sujetos antes del encuentro con ellos. Pretendemos construir una institucionalidad con los sujetos que viven cotidianamente en el territorio en articulación con el Estado, pero más allá de la estructura del Estado. Este modo de intervención colectiva ubica a los trabajadores también como sujetos, planteando un encuadre que implica llevar adelante una planificación en situación (Equipo puente de tierra, 2009, s/p).

Las actividades de juego se desarrollan en "la canchita"; una especie de plaza (terreno vacío) que resiste a la sobrepoblación de casillas en un extenso asentamiento ubicado a lo largo de las vías del tren en Rosario. Esta villa se caracteriza por el crecimiento poblacional 
en los últimos años, una pirámide poblacional joven, precariedad habitacional, el desempleo o subocupación, y la labilidad en el acceso y uso efectivo de las instituciones público estatales. La población que vive allí no accede a los servicios básicos: las conexiones de agua y luz eléctrica son precarias y la recolección de residuos es irregular ya que los camiones acceden solo parcialmente y se forman basurales. La eliminación de excretas se da en pozos negros, hoyos o excavaciones no viables de desagotar. La acumulación de agua de lluvia impide la circulación por las callecitas de tierra (algunas son, apenas, pasillos estrechos). Las manzanas no están definidas. En su gran mayoría las viviendas son construcciones poco sólidas con bloques, chapa y cartón.

El territorio es el punto de partida para el encuentro entre instituciones estatales y con la población, para el trabajo conjunto y para lo que deviene proyecto Puente de Tierra. El dispositivo promueve un habitar democrático del espacio común y propone el reconocimiento mutuo en el encuentro con los/as otros/as (diferenciándose de un discurso que sabe del otro antes del encuentro). Los actores entran en escena en un intercambio con características especiales: se hace hincapié en la posibilidad de construcción en conjunto y en propiciar relaciones de igualdad, dignidad, cooperación, confianza y respeto de las singularidades (incluidas la de cada trabajador/a del equipo). Se trata, además, de un vínculo diferente del que instalan las instituciones por si solas.

Respecto a la inscripción de Puente de Tierra en el marco de las políticas públicas territorializadas, es preciso destacar que los modos de funcionamiento de cada una de las instituciones territoriales difieren entre sí (se encuadran en distintos objetivos, ritmos y formas de vincularse con la población). A su vez, estos modos fueron modificándose des- de el inicio de la experiencia en el año dos mil nueve a su cese en dos mil dieciocho. Participan del dispositivo instituciones dependientes de tres secretarías municipales distintas (Desarrollo Social, Salud Pública, Cultura); los centros de salud intervinientes pertenecen a distritos diferentes; y, además, se suman las particularidades de la Universidad Nacional de Rosario (UNR). Las lógicas de la política y la gestión de las diferentes secretarías difieren en relación a: la población usuaria (universalidad - focalización); la contratación de los/ as trabajadores/as que incide en la permanencia de los servicios que presta; el objetivo que prima (siendo que Desarrollo Social propone una lógica de corte más promocional y socioeducativo, y en la Secretaría de Salud prevalece lo asistencial); y las burocracias que construye (servicios y procedimientos en el caso de salud; dispositivos y talleres puntuales y aislados en cultura; y cambios permanentes en el perfil de instituciones de Promoción Social).

Dichas particularidades de la política social y de salud inciden en los procesos de trabajo del equipo. Es claro que algunas modificaciones definidas desde niveles centrales impactan negativamente en la posibilidad de dar continuidad al trabajo a lo largo del tiempo. Además, en los diarios de campo de las reuniones se leen reflexiones del equipo respecto al impacto de los procesos de descentralización en la esfera política de la ciudad que inician a mediados de la década del noventa. Se trata de una tensión y contradicción entre la mayor autonomía de los/as trabajadores/as para desplegar una institucionalidad efectiva en el ejercicio de derechos de amplios sectores, con la contraparte de su matriz neoliberal respecto al desmonte del Estado, en la que el diseño de la política termina siendo responsabilidad de los equipos locales.

La presencia estatal en las barriadas populares de Rosario se caracteriza, por un lado, 
por las instituciones que aún resisten al empobrecimiento de las políticas sociales (fundamentalmente escuelas, centros de salud y centros de convivencia barrial); y, por otro lado, en términos punitivos, se agudiza otra característica de la presencia estatal con el despliegue en aumento durante los últimos años de las fuerzas represivas (policía, gendarmería, policía de acción táctica).

De esta manera, se desarrolla una paulatina retirada del territorio por parte de los equipos de las instituciones. Respecto a lo cual destacan dos motivos, por un lado, las dificultades del trabajo en relación a la intervención en situaciones de vulnerabilidad de las poblaciones teniendo en cuenta una falta de recursos estructural; $y$, por otro lado, el hecho de que los dispositivos o talleres en territorio, orientados en una línea preventiva, no han sido la prioridad en la planificación de los proyectos institucionales. Tanto para la gestión como hacia el interior de los equipos, tal vez por esa influencia, la valoración de este tipo de espacios pareciera ser menor respecto a las actividades que se llevan a cabo puertas adentro. A pesar de que a nivel municipal tanto las políticas de la Secretaría de Salud Pública respecto a la atención primaria de la salud, como las políticas de Promoción Social y Cultura, tienen fundamentalmente una base de inserción territorial; se plasman las hegemónicas demandas de atención por parte de salud, y de trabajo de oficina por parte de Promoción Social.

La incidencia de este escenario en el despliegue de Puente de Tierra representa una amenaza de subsistencia del dispositivo, generando concretamente que algunos/as miembros del equipo, afectados por decisiones de las gestiones intermedias, no continúen participando; y, además, contribuye a generar un clima de tensión y descontento con otras situaciones que se suman a la serie dificultan- do la fluidez del espacio. Un ejemplo de estas últimas es la falta de presupuesto específico para materiales o actividades; ya que recurrir a los recursos de cada institución genera diversos conflictos.

A raíz de todos estos obstáculos, es posible pensar que el sostén del Puente de Tierra tiene variados contrapuntos respecto a lo que han sido, durante los últimos años, los lineamientos de la gestión municipal.

\section{Trabajadores / as como Unidad de Tra- bajo en cogestión}

El cotidiano del mundo del trabajo se sitúa como una micropolítica, en la que el/la trabajador/a es, individual y colectivamente, productor/a y producido/a, en los modos de actuar y los procesos relacionales. (Franco; Merhy, 2009) Así, se evidencia que los/ as trabajadores/as son actores/sujetos de la acción. Esta conceptualización se diferencia radicalmente respecto a visualizar el mundo del trabajo como un mundo donde el/la trabajador/a no puede hacer nada, por estar totalmente capturado en las estructuras que lo definen y determinan.

A los fines de indagar sobre los procesos de trabajo del equipo interinstitucional e interdisciplinario en una experiencia territorial, se adscribe a una perspectiva que incluye una manera cartográfica de analizar el funcionamiento de organizaciones de salud o unidades de salud. Partir de los actores/sujetos en acción, en tanto artífices del cotidiano de esas unidades, permite focalizar en los procesos de trabajo constructores de las prácticas de salud, observados en sus dinámicas productoras del cuidado, y en la producción de esa cotidianeidad, que es múltiple, relacional y situada.

Las unidades de salud tienen un modo de 
producción dependiente del trabajo vivo en acto que por sí solo aporta una alta complejidad a la escena, pues este trabajo vivo, al ser operado en acto en los procesos de trabajo, contiene una gran potencia instituyente para formar redes, con alta capacidad de subjetivación (Franco; Merhy, 2009). En el marco de estas categorías, el equipo que lleva adelante Puente de Tierra es considerado como una unidad de trabajo a pesar de que su práctica territorial y la pertenencia institucional de sus miembros no se circunscriban al ámbito de la salud. De esta manera, si bien el equipo Puente de Tierra no se constituye como una organización de salud en función de los objetivos que erige el proyecto, su práctica se desarrolla operando en el campo de los procesos de subjetivación y produciendo ciertos procesos de cuidado.

Respecto del carácter intersectorial y público de la experiencia Puente de Tierra se retoman los aportes de Rosana Onocko Campos (2002) quien, refiriéndose a organizaciones de servicios públicos (no lucrativos) de salud, desarrolla una discusión sobre la gestión apelando a su dimensión productiva y generadora. Dicha dimensión es resaltada en el concepto de cogestión, como síntesis siempre provisoria entre la tentativa de la heterogestión taylorista y la utopía autogestionaria. La gestión clásica trabaja la dimensión de administrar. Es la disciplina del control por excelencia preocupada con el aumento de la producción, de plusvalía, productividad y reproducción del status quo.

Ante la pregunta sobre cómo otorgar a la gestión, entonces, un desarrollo de su dimensión creativa, Onocko Campos (2002) señala:

La primera cuestión sería que instauremos para la gestión un lugar y un tiempo. Crear e instituir espacios en los cuales se pueda experimentar la toma de decisiones colectivas. Espa- cios en los cuales sea posible formular proyectos.

Espacios que puedan transformarse en instancias, (Lagache \& Pontalis, 1992) en el sentido de procesar aquello que les llega, y tener un grado mayor de análisis de la implicación con aquello que producen (p.2).

Respecto a la forma de gestión que despliega el equipo de Puente de Tierra resalta la dimensión productiva en las instancias de debate y decisión colectiva.

\section{Síntesis del proceso de trabajo del equipo}

El estudio exhaustivo sobre los procesos de trabajo del equipo de Puente de Tierra desde octubre 2012 a diciembre 2014 se desarrolló desde la sistematización de experiencias, utilizando los procedimientos de: recuperación del proceso vivido (reconstrucción de la historia y ordenamiento de la información); y reflexión posterior (Jara, 1994). Se aborda la experiencia separándola en períodos anuales: 2012, 2013 y 2014. En función del amplio material de registros en diarios de campo se ordena la información en los siguientes cuatro puntos:

I- Conformación y modificaciones del equipo Puente de Tierra. Participación de otros actores (comunitarios $\mathrm{y} / \mathrm{o}$ institucionales).

II- Organización del trabajo del equipo en reuniones y actividades en territorio. Frecuencia y dinámica de cada una.

III- Logros. Aspectos y/o actividades valoradas positivamente.

IV- Temas y/o situaciones problemáticas respecto al desarrollo del trabajo del equipo. Debates, y acciones. 
Respecto a los elementos centrales de dichos procesos se lleva adelante un ejercicio de análisis, síntesis e interpretación crítica. Con el objetivo de introducir una visión global de los acontecimientos más importantes, se plasma un recorrido que explicita el sentido de la experiencia, valora los elementos que han intervenido e identifica las modificaciones e insistencias a lo largo de todo período estudiado. Asimismo, se utilizan como guías los puntos que refieren a los componentes destacados.

El equipo de adultos/as que participan en carácter de coordinación es relativamente estable y se conforma por diferentes instituciones de la zona ${ }^{4}$ y la Universidad Nacional de Rosario (UNR), a través de la participación de estudiantes que realizan residencias de grado y posgrado. Si bien desde el comienzo permanece la mayoría de instituciones, los profesionales participantes fueron modificándose. Del total de integrantes del equipo (entre nueve y doce) aproximadamente tres fueron cambiando año a año. Si bien esto supone cierta continuidad y estabilidad de un grupo de siete personas, es un punto conflictivo que reedita la tensión mencionada, entre la permanencia de Puente de Tierra y los lineamientos de las políticas públicas. Se destacan las dificultades con las instituciones de pertenencia de los/ as participantes y respecto a la necesaria estabilidad del equipo para la construcción del vínculo con los/as chicos/as, el equipo y el resto de la comunidad.

La participación de otros actores, tanto de carácter comunitario como institucional es ineludible respecto a los objetivos explícitos del proyecto de Puente de Tierra para la construcción de redes y vínculos. A lo largo del proceso de trabajo, fundamentalmente du-

\footnotetext{
${ }^{4}$ Se omite la referencia explícita por razones de resguardo de información.
}

rante los años 2013 y 2014, se profundiza la estrategia de trabajo en este sentido. Para ello, se organizan mayor cantidad de actividades junto a vecinos/as del barrio; y se incorpora la participación de una persona adulta de la comunidad en las actividades de juego en la villa. Ante la difícil tarea de sostenerse en un territorio con conflictividad y violencia social creciente, los vínculos con la comunidad se instalan a la manera de un resguardo para el equipo y el dispositivo mismo. Por otro lado, durante el año 2014 el equipo define dedicar más tiempo a las tareas de reflexión/planificación y re-trabajo. Así, en el marco de un nuevo espacio-otro mensual, en agosto de ese año es convocada una psicoanalista a modo de actor externo. Este punto se retomará luego, para interrogar las implicancias de la participación de estos otros/as.

En cuanto a la organización del trabajo del equipo, a grandes rasgos, consiste en dos tipos de actividades: por un lado, reuniones destinadas a debatir, planificar y evaluar el desarrollo del dispositivo, y por otro, las propuestas lúdicas en territorio. Desde el primer período estudiado se observan dificultades en ambas actividades. Las reuniones de equipo son desordenadas e insuficientes, tanto para tomar decisiones ante situaciones problemáticas como para planificar los juegos que resultan improvisados y a cargo de solo algunos miembros del equipo, generando malestar.

A raíz de este diagnóstico, a lo largo del proceso de trabajo, el equipo realiza una serie de modificaciones: incorporación de registros escritos de las reuniones para abonar a la planificación y organización de los debates y mejorar la comunicación interna; distribución de tareas con duplas de trabajo encargadas de coordinar las actividades lúdicas en territorio y preparar previamente los materiales necesarios para las mismas; profundización del debate sobre los roles de cada miembro del 
equipo; optimización del tiempo dedicado a las actividades y según horarios acordados; reorganización de los bloques de actividades en territorio y las reuniones en tres encuentros lúdicos y uno de reunión de planificación/ evaluación, sucesivamente; incorporación de un encuentro mensual de reunión adicional, a modo de momento reflexivo sobre Puente de Tierra y; convocatoria a una persona externa al dispositivo.

Así, paulatinamente las reuniones son más ordenadas y se dispone de tiempo para la planificación, debate y toma de decisiones. Sin embargo, subsisten dificultades para sostener las actividades en territorio, entre otros motivos, por problemas en la organización del trabajo.

Por otro lado, los aspectos valorados positivamente por parte del equipo de trabajo se vinculan directamente con los objetivos de Puente de Tierra en cuanto a la centralidad de la actividad lúdica con los/as niños. El desarrollo cotidiano de los juegos en la villa configura un aspecto gratificante de la tarea colectiva. En los registros de campo se lee cómo son valoradas las respuestas que ha ido construyendo el equipo ante las dificultades de organización y planificación para el desarrollo de las propuestas de juego. La capacidad de convocatoria para la participación de gran cantidad de niños/as, con nuevas incorporaciones a partir de cumplir los diez años de edad, también es un aspecto positivo. A lo largo del proceso de trabajo el equipo evalúa, además, estar atravesando un buen momento en la relación y las experiencias conjuntas con organizaciones barriales, ONG e instituciones público-estatales con presencia en el territorio. También resalta la valoración positiva respecto al bagaje de experiencias y las producciones a lo largo de la historia del dispositivo y la posibilidad de transmisión hacia el interior del equipo, los/as niños/as y el resto de los actores comunitarios.

Respecto a los temas/problemas en relación al desarrollo del trabajo, además de los ya mencionados; cobran centralidad las situaciones conflictivas en el territorio. En los fragmentos de registros de campo resalta el relato de tres escenas por las cuales se vio afectado, de una u otra manera, el desarrollo del Puente de Tierra: por un lado, un episodio sucedido minutos antes de empezar la jornada, protagonizado por algunos jóvenes que muestran armas de fuego e increpan a un miembro del equipo respecto a una situación ajena al dispositivo, y, por otro, dos escenas transcurridas durante las actividades lúdicas (la interrupción por parte de un niño que habitualmente participa del Puente de Tierra que, reactualizando en el seno del grupo un gran enojo por situaciones injustas sufridas en su historia singular, se llevó los materiales de juego, y no se pudo continuar la actividad general, por un lado y; por otro, la irrupción de algunos jóvenes durante el juego de los/as más chicos/as, que derivó en una situación de pelea y discusión).

De este modo, la apuesta por parte del dispositivo a construir vínculos y puentes atraviesa escenarios de turbulencia y complejidad. Cobran espesor los fragmentos del documento colectivo inédito, ya que de esto se tratan las "condiciones propias de un territorio donde prima la ruptura del lazo social" (Equipo Puente de Tierra, 2009, s/p). El encuentro en territorio confronta al equipo con situaciones de extrema vulnerabilidad y violencia que, a lo largo del proceso de trabajo se enuncian como episodios conflictivos, complejidad de la situación de algunas familias, conflictividad creciente en el territorio, situación de violencia a nivel social, exposición de personas del equipo, enfrentamientos, peleas, tiroteos, fragmentación, falta de legalidad u otro tipo de legalidad, crudeza de las situaciones, injusticia, límites de la palabra, etc. Luego se reto- 
mará este punto para articular una conceptualización sobre tales enunciados y situaciones problemáticas, así como sobre las respuestas por parte del equipo, que se analizan a continuación.

Como parte del proceso de trabajo se desarrollan debates y decisiones como respuestas ante aquellos temas y/o situaciones problemáticas. Respecto a las primeras dos escenas referidas, inmediatamente se asume el contexto de vulneración por parte de las personas involucradas, a la manera de un sabemos que no es justo; se apela al diálogo y al vínculo previo; y en una de ellas también participan otros actores (vecina). En cambio, frente a la escena de pelea, el equipo parece no dar acción inmediata como respuesta, experimenta un límite a la palabra y en un momento posterior, a partir de la reflexión, se abre la posibilidad de generar criterios para la acción futura en posibles situaciones similares.

En relación a las situaciones conflictivas en territorio, el equipo subraya la necesidad de contar con un resguardo para sostenerse: resguardo del colectivo de trabajadores/as, del dispositivo Puente de Tierra y de cada miembro. Es posible resaltar dos movimientos realizados para abordar esta situación. Un movimiento es dirigido hacia adentro, tiene el objetivo de consolidar el colectivo y se lleva a cabo organizando y dividiendo las tareas y fortaleciendo criterios y decisiones comunes. Otro movimiento se da hacia afuera, convocando a otros actores a participar (vecinos, organizaciones barriales, ONG, comedores comunitarios, universidad, una psicoanalista, etc.).

Por otro lado, en los registros de campo de las reuniones de equipo se enuncia el tema/problema de que "algunas situaciones que se dan en el territorio imponen límites a la palabra y llevan a poner el cuerpo". Respecto a lo cual aparece en clave de respuesta, durante el período 2014, un elemento novedoso (en tanto es expresamente señalado). Se trata de una propuesta de abordaje del problema en las actividades lúdicas, con los/as chicos/ as, apostando a la palabra como herramienta para elaborar ficciones sobre escenas cotidianas y a la puesta en juego del cuerpo a través de la expresividad y el deporte.

En cuanto a la problemática respecto a la falta de financiamiento de materiales necesarios para las actividades, es planteada desde el primer período estudiado e insiste sin resolución durante los tres períodos, a pesar de las gestiones que el equipo emprende al respecto.

Por último, en las respuestas que el equipo plantea frente las dificultades de la práctica, el hilo conductor son las instancias de debate y construcción colectiva. Allí adquiere especial importancia el ejercicio reflexivo sobre el propio trabajo, los obstáculos, los objetivos y las acciones a llevar a cabo.

Las dificultades que implica el trabajo en Puente de Tierra se presentan como una marea donde el equipo, por momentos, parece naufragar. Sin embargo, es posible señalar que, en el despliegue cotidiano, aprende a navegarla. Interesa, igualmente, sondear los modos en que estas dificultades afectan al equipo.

\section{Encerronas cotidianas. Con toda la mar detrás}

Ulloa (2011) señala que "Con toda la mar detrás" es el nombre de una canción sobre un naufragio, y usa esta expresión

para aludir a la actitud positiva de los integrantes de un equipo asistencial, docente o de cualquier naturaleza, que se mostraban propicios a debatir -críticamente y a la manera de un ensayo- su futuro accionar. Resultaban acreedores de este elogio, que alude 
a la fuerza colectiva que los impulsa y respalda. (p.17)

Por lo mismo, en el título de este escrito, se asocia al equipo en juego en Puente de tierra la misma fuerza, necesaria para enfrentar las circunstancias adversas del escenario de la numerosidad social, donde transcurren los hechos. Además, esa canción habla de un naufragio donde los marineros que tripulaban la nave, que eran pescadores desde antes de nacer, no sabían nadar. La canción dice que el destino no les quiso enseñar. Sin embargo, Ulloa sitúa allí las arbitrariedades del poder, que no solo no les enseñó a nadar como elemento esencial de seguridad de los pescadores, sino que de esas arbitrariedades están llenos los escenarios de la numerosidad social. Esta idea remite aquí, también, a una postura crítica, política, y a la desnaturalización consiguiente, frente a las adversidades en nuestro ámbito.

Entonces, ¿en qué consisten los modos de trabajo en equipo? ¿De qué se tratan esas construcciones colectivas? ¿De qué se tratan esos reparos? ¿Qué elementos esenciales de seguridad/resguardo/cuidado/reparo, a la manera del saber nadar de aquellos pescadores, es posible ubicar en el trabajo del equipo del Puente de Tierra?

La noción de reparo remite a: restauración; advertencia, nota, observación sobre algo, especialmente para señalar en ello una falta; duda, dificultad o inconveniente; o bien, a lo que se pone por defensa o resguardo. A este concepto se asocia la palabra amparo, cuya definición convencional, a su vez, nos remite a la acción y efecto de amparar o ampararse; y a la persona o cosa que ampara. (Real Academia Española, 2019)

Significativamente es posible ubicar en los procesos de trabajo del equipo del Puente de Tierra, movimientos relacionados a la discon- formidad del orden existente, al reparo (darse cuenta) de las dificultades inherentes y al ejercicio de amparar-se para construir amparos, y/o constituir-se como amparo.

Ahora bien, antes de profundizar sobre los modos y capacidad de elaboración o respuesta del equipo, es precisa una indagación sobre lo que se da en llamar desamparos sociales y subjetivos, vulnerabilidades, violencias, crueldad, etc.

Se toma entonces como referencia el concepto de encerrona trágica de Ulloa (1995) ya que las conceptualizaciones sobre la ternura, que luego propone, tienen como telón de fondo el horror de la represión, la marginalización y el escándalo de la miseria. El autor desarrolla dos ideas particularmente útiles para trabajar con afectados en grados y situaciones distintas; son lo que sitúa como encerrona trágica y como efecto siniestro. Si bien dichos conceptos han sido extraídos de su quehacer en el campo de los Derechos Humanos; los diversos organismos e instituciones que se ocupan de la vida cotidiana de la gente están atravesados por la trasgresión a estos derechos.

El paradigma de la encerrona trágica es la tortura, situación donde la víctima depende por completo, para dejar de sufrir o para sobrevivir, de alguien a quien rechaza totalmente. Otro tanto acontece con sus familiares.

La tragedia así concebida es una situación de dos lugares, opresor-oprimido, sin tercero de apelación. Esta falta absoluta de una instancia para apelar, tal como ocurre en el terrorismo de estado, da a la situación el carácter de encerrona concreta y psicológica. (...) De cualquier manera, es sobre la invalidez extrema de tal situación que la extorsión se ejerce. 
También he señalado en otros trabajos cómo esa falta de tercero de apelación, realidad frente a la cual se encuentran los familiares, sin puertas que golpear en los momentos de terrorismo de estado, fue uno de los orígenes de los organismos de Derechos Humanos, cuando los afectados se agrupaban y organizaban constituyendo ellos mismos una instancia de apelación. (Ulloa, 1995, p.133)

En su análisis sobre el poder efectivo y moral de los organismos de Derechos Humanos para romper con aquella encerrona, Ulloa (1995) señala una función importante que resulta ser una salida: la de impedir que los crímenes se secreteen (secuestros y desapariciones durante la dictadura militar, muestran y ocultan simultáneamente; sostienen un siniestro secreto a voces).

El psicoanálisis ha estudiado esta situación mostrando cómo el secreto oculto del cual se desprenden indicios, tiende a promover el efecto siniestro, a la sombra de la renegación de los hechos, sin poder ocultar el temor y la parálisis resultantes de lo que siendo atroz, permanece semioculto. Se niega que se niega, como una defensa muy elemental de alguien que intenta ocultar lo temido, o tal vez pretende vanamente ocultarse de aquello que lo atemoriza. (...) superar tanto la renegación como el efecto siniestro implica el duro trance de enfrentar la tragedia cruda, que por permanecer semioculta, mantenía y mantiene aún, aunque atenuada, su eficacia. (Ulloa, 1995, pp.133-134)
Ahora bien, en las instituciones públicas asistenciales, y en menor medida en las educativas, el factor trágico se multiplica al desenvolver sus actividades contextuadas en el escándalo de la pobreza y con comunidades mortificadas. En estos ámbitos, donde lo establecido (instituido) toma la rigidez cultural de la mortificación, la tragedia en forma de encerrona trágica es un factor epidemiológico habitual y etiopatológico central para un abordaje de la psicopatología social. (Ulloa, 1995)

Es claro que las encerronas trágicas afectan no solo a las poblaciones directamente implicadas en la marginalidad, sino también a quienes, por medio de instituciones, se ocupan de su asistencia. Sin embargo, en relación con el ámbito institucional, los efectos dolorosos (dolor psíquico) de la encerrona no siempre son advertidos.

Es posible articular el concepto de encerrona trágica y la manera en que impacta en los diferentes ámbitos sociales con los procesos de trabajo del equipo Puente de Tierra. Lo desarrollado en el punto sobre las situaciones/temas problemáticos del trabajo y la conflictividad en el territorio, constituye la forma en que el equipo vivencia y expresa las diversas encerronas trágicas de su cotidianeidad y la del barrio. También es posible pensar que el equipo es testigo amenazado por ese matiz del sufrimiento social que es la cultura de la mortificación (Ulloa,1995). Sin embargo, el equipo todavía no ha zozobrado ${ }^{5}$. Con toda la mar detrás, respecto al proceso de trabajo del equipo, no ha desaparecido la producción de pensamiento ni el suficiente valor para resistir. Por mantenerse sensible y en busca de "una salida", el equipo de Puente de Tierra no está del todo sumergido en la mudez sorda y ciega de la mortificación.

\footnotetext{
${ }^{5}$ Resulta significativa la asociación del término zozobrar que remite a naufragar.
} 


\section{Terceridad de apelación. Gestión de utopías}

Para superar tanto la renegación como el efecto siniestro el equipo atraviesa el trance de enfrentar la tragedia y apela a instancias de debate y reflexión que ponen en palabras en ámbitos colectivos -y no secretean- las situaciones de extrema violencia y vulnerabilidad que se dan en el territorio. Además, el hecho de que el equipo de trabajo se asuma implicado como sujeto de su propia práctica, así como el carácter instituyente con el que se presentan los procesos de trabajo en relación al trabajo vivo en acto se incluyen como elementos que rehúyen de la coartación subjetiva que impera en las encerronas trágicas.

En torno a la pregunta sobre los modos en que el equipo enfrenta las mentadas tragedias y desamparos, también es pertinente analizar cómo entra en juego en Puente de Tierra el tercero de apelación, que al contrario desfallece en las escenas de encerrona. En primer lugar, es importante subrayar que el equipo de adultos se constituye como tercero de apelación hacia los/as niños/as con los/as que trabaja. Encontramos un claro ejemplo en la escena que protagoniza el niño que despliega en el seno del grupo la actualización de escenas de injusticia de su historia de vida. Para la intervención en el conflicto los miembros del equipo cuentan de un punto de facilidad relativa (Ulloa, 1995) respecto al niño, por su condición de adultos; sin embargo, experimentan cierta paralización ante esa escena. A raíz de situaciones como ésta el equipo decide profundizar la convocatoria a la participación de otros actores de carácter comunitario y/o institucional. Entonces, es posible afirmar que dichos actores, cuya participación es enunciada en términos de resguardo, se constituyen a la manera de terceros de apelación.

Ante situaciones sin el respaldo institucio- nal necesario -es decir, una política pública que dé respuesta a los escándalos de la miseria-, el equipo de Puente de Tierra se agrupa y organiza constituyendo ellos mismos una instancia de apelación. Como vimos, esa fue la manera en que surgen los organismos de derechos humanos cuando los familiares de las víctimas experimentaron la falta de tercero de apelación, sin puertas que golpear en los momentos de terrorismo de Estado.

Además, el equipo de trabajo apela nuevamente a una terceridad que supere la encerrona cuando plantea la necesidad de ser escuchado por alguien desde otro lugar para pensar otra perspectiva sobre las problemáticas. A los fines de sortear los efectos de encerrona en la escena trágica, que paralizan el entender, el equipo demanda ser escuchado desde un punto clínico de facilidad relativa, sin estar ajeno al campo, pero tampoco en el centro de la escena.

Por otro lado, la utopía se constituye como otra operación posible para superar la renegación, en el sentido que Ulloa (1995) le asigna:

en su forma más actualizada, un sentido que se expresa en un negarse a aceptar aquello que niega (encubre) las causas más arbitrarias de los sufrimientos individuales o colectivos. Si estas arbitrariedades están veladas, lo están por un proceso de renegación (negar que se niega) con que la víctima asume su mortificación y la desglosa de lo que la origina.

La utopía como operación clínica supone una doble vuelta: la de una negación con sentido positivo (negarse a aceptar aquello que niega lo subyacente), opuesta a la propia de la renegación.

El pasaje de la mortificación idiotizante a la toma de conciencia de la trage- 
dia supone -éste es el problema- la recuperación del sufrimiento embotado por la mortificación, en todo caso por su carácter mortecino, apagando la conciencia. Éste es el obstáculo que hace tan difícil superar la alienante anestesia mortificada. (p.188)

¿Es posible, entonces, ubicar esta negación con sentido positivo en los procesos de trabajo del equipo que lleva adelante Puente de Tierra? A lo largo del análisis de las respuestas que el equipo ensaya frente a las dificultades de la práctica en territorio, se han señalado diversas decisiones y debates a la manera de movimientos que posibilitan señalar un proceso de toma de conciencia de situaciones trágicas. Además, a partir de experimentar dichas situaciones, el equipo enuncia cierta afectación que es posible situar como la recuperación del sufrimiento que en situación de encerrona quedaría embotado. En los registros de campo de las reuniones de equipo se encuentran estas expresiones: "la crudeza de las situaciones en la villa nos pone mal"; "nuestro objetivo es jugar, pero son dos horas que cansan más que cualquier otro trabajo. Es como una piedra pesada y terminamos agotados/as."; "lo que nos pasa es que sentimos dolor por la pobreza y la injusticia que impera en el barrio. También enojo.”.

Ulloa (1995) profundiza sobre el valor de la utopía en tanto actividad crítica promotora de crisis y que posibilita que lo repudiado en la renegación no retorne como malestar quejoso, sino que se instale una doble denegación positiva: la negación a aceptar todo aquello que niega (repudia) las verdaderas causas de una situación mortificada.

La utopía, entendida como acción develadora actual, mantiene algo del antiguo linaje heroico del término, $y$ pone en juego todos los recursos disponibles, sin demora quejosa frente al auxilio que no llega, aun cuando se lo siga reclamando. Es así que surge, junto a la utopía actual, el otro pilar indispensable para toda refundación: la autogestión, quizá también un horizonte de la utopía.

Utopía y autogestión son dos requisitos valiosos para que lo nuevo se establezca. (Ulloa, 1995, p.165)

\section{Y, sin embargo, JUGAR. Equipo en Juego}

Como característica específica, el equipo de Puente de Tierra es un grupo de adultos/ as en juego, llevando adelante actividades de recreación con niños/as y jóvenes. Aquí, recrear evoca al menos a dos sentidos: uno en tanto actividad lúdica, lo que divierte, lo que entretiene; otro, ligado a la creación, lo creativo, el hecho de crear algo a partir de lo existente y, de alguna manera, a la invención. Recrear, entonces, corresponde a posibilitar que la pregunta por las infancias y las juventudes permanezca abierta a la pluralidad de sentidos, a las transformaciones, a las singularidades, a la huida de los lugares comunes y, fundamentalmente, al juego.

Las actividades que se desarrollan son variadas: serigrafía, juegos con pelotas, lectura y escritura de historias, panificación y venta de lo producido para los paseos, puestas en escena, dibujo, adivinanzas, juego de postas, cuidado de la plaza a partir de plantar árboles, juegos de mesa, videos, etc. El eje transversal de todas ellas es el juego, el jugar; una primera manifestación del vivir creador que luego hará posible la experiencia cultural (Winnicott, 1972) y que posibilita una articulación simbólica de lo real, de lo pulsional. El juego 
como lenguaje primordial de los/as niños/as; que pretende ser elaborativo y subjetivante.

Ahora bien, es necesario plantear un interrogante en torno a las formas que se da el equipo de sostener, reactualizar y ejercitar como adultos/as, en tiempos de conmoción social, la asimetría necesaria respecto a los/ as niños y jóvenes con los/as que trabaja; ya que se trata de subjetividades en vías de constitución (Zelmanovich, 2003). Las personas adultas se constituyen, a la manera de pantalla protectora, como mediadores con la realidad que se presenta signada por el desamparo social y subjetivo; ya que la falta de recursos para subsistir refiere no solo a la falta de comida, techo, salud, etc. como falencia material; sino, además, a la fragilidad e inconsistencia de muchos de los discursos que sostienen el vínculo social. Entonces, esta realidad también vulnerabiliza a los/as adultos/as en juego, aunque de diferente manera. Equiparando la vulneración de los/as adultos/as a la de los/ as niños/as y jóvenes se correría el riesgo de dejar de ofrecer mediaciones necesarias para significar la realidad, o derivar asimismo en la culpabilización, patologización o criminalización de los/as más pequeños/as.

Zelmanovich (2003) sostiene que "La pregunta que cabe formularnos es: ¿cómo lograr no transferir la propia vulnerabilidad al niño o al joven?” (p.11) y explicita algunas respuestas posibles. En primer lugar, se trata del trabajo entre varios o tras bastidores, donde la constitución de un nosotros, donde comparten las tareas diferentes adultos/as, surge como oportunidad para construir una nueva narrativa colectiva. "Algo así como 'adultos con adultos', al amparo de los niños y los jóvenes." (p. 12) Esto es un modo de atender no solo al desamparo del sujeto infantil, sino también de los/as adultos/as. En segundo lugar, se delinea el reparo como la apuesta a sostener el lugar de adultos/as mediadores con la sociedad y la cultura y el hecho de proponerse y estar dispuestos a constituir para los/as niños/as y jóvenes aquellos Otros disponibles que puedan ejercer funciones subjetivantes.

En Puente de Tierra ambas cuestiones se sitúan claramente. Por un lado, la experiencia se despliega desde un equipo que configura colectivamente un recinto para pensar, apelar y sostener/se el trabajo mutuamente. Por otro lado, en los fundamentos mismos de la práctica se despliegan las características subjetivantes del dispositivo (jugar, participar cada uno/a a su manera, producción de vínculos de pertenencia, sentido e identidad, etc.). De tal forma, lo colectivo se convierte en un marco donde se llama al sujeto a tomar un lugar (Grande, Mana, \& Zampiero, 2018). Puente de tierra se trata de adultos/as que están en función, que se abocan a la tarea de resguardar a los/as niños/as y jóvenes con quienes trabajan respecto a la posible re-vulneración. Estos adultos, además, se instalan como facilitadores/as al acceso a la cultura, y se ocupan de generar condiciones necesarias para la creación cultural colectiva, que es una vía regia hacia aquel acceso.

Ahora bien, en las actividades recreativas, el juego es el centro de la propuesta de Puente de Tierra; y el equipo se dispone, entonces, a jugar. ¿Qué características son necesarias para jugar? ¿Surge aquí otra manera en que el equipo lleva adelante la elaboración de desamparos y de lo trágico?

Si bien el escenario de la canchita puede parecer cotidiano para los/as niños/as y jóvenes, los rituales de comienzo, armado, desarrollo y final de la actividad en Puente de Tierra permiten y/o intentan instaurar otro momento y otro espacio. Se introduce aquí la noción de otra parte que permite que el/la niño/a se abstraiga y que promueve procesos subjetivantes. Puntualmente, la abstracción de la que hablamos se articula con suspender (también elabo- 
rar, de alguna manera) la cruel situación de pobreza y violencia que se vive en el barrio; y que sin embargo insiste cotidianamente en el espacio mismo del dispositivo. Numerosas veces se presencian escenas de riñas, heridos de cuchillazos, relatos sobre consumo abusivo de drogas, prostitución, abandono o maltrato infantil, hambre, tiroteos, etc. En estos contextos tan duros, el dispositivo intenta una y otra vez hacer lugar a las historias singulares de los/as niños/as, cuidando su cualidad de niños/as y recuperando sus saberes y experiencias previas. El registro y el trabajo sobre la crueldad, y la ternura posible, hacen que el espacio se mantenga en pie.

Además del jugar, entran en la escena los cuentos, las canciones, el teatro, la impresión con serigrafía, el dibujo, el deporte, el humor, las historietas, etc.; todas estas son actividades ligadas a lo creativo y la ficción. Es pertinente aquí hilvanar los conceptos de Ulloa (2011) respecto al desarrollo del humor conjetural y la resignificación, herederos de la ficción infantil que, a través de la producción lúdica, se propone no renegar los hechos de la realidad. Se trata de una actividad imaginativa que conlleva el grado de inventiva que supone resignificar los hechos. El humor, como una forma de la valentía, es un fluido capaz de penetrar las rigideces de lo real.

Prosiguiendo con la aplicación del concepto hacia los equipos en los que Ulloa (2011) opera, él mismo señala que:

Cuando un equipo asistencial (...) se hace fuerte en el per-humor que conjetura salidas, promueve la salud mental como producción cultural capaz de decidir acciones y, a su vez de accionar sobre ellas, en sentido per-sistente, con una intensidad sostenida en el tiempo. (p.127)
Además, el autor relaciona el per-humor con un

humor inclaudicable, hasta el final, el necesario para ser testigo, cualquiera sea el oficio desde el cual resulte impulsado el accionar quijotesco y donde a la utopía del Quijote venga a sumarse la sensatez inquebrantable (...) de un Sancho Panza representando a un Sancho Pueblo. (p.127)

Significativamente, Don Quijote de La Mancha, el famoso caballero de la novela de Miguel de Cervantes Saavedra, luchaba contra toda injusticia.

De la mano de diversos artificios, el equipo en juego instala un territorio poético, artístico, literario, de ficción. Vienen a este territorio diversos juegos, personajes, canciones y actividades. Así, una de las propuestas más relevantes es un juego donde se erige un mítico "Dibujante de la Noche" con quien los/ as chicos/as se comunican a través de cartas. A raíz de estos intercambios nace una serie de historietas sobre el barrio y sus problemáticas, que relata las aventuras de "Super-Villa", un vecino que se convierte en héroe por accidente y ayuda a su querido barrio.

Año tras año el mítico personaje Dibujante de la Noche intercambia cartas con los/ as niños/as y jóvenes del Puente de Tierra; a los/as que en diversas oportunidades les pide que le diseñen un traje, o que inventen otros personajes con poderes mágicos, etc. De esta manera, las historias y personajes se van sucediendo, con un "continuará" al final, que sostiene la intriga hasta la próxima noticia del Dibujante de la Noche. Con la misma dinámica nace "Puentechueka", la heroína del barrio, que toma las características de los/as héroes y heroínas que los/as niños/as habían dibujado durante las actividades. 
En estas recreaciones humorísticas, solapadamente, entran en juego personajes que toman algunos rasgos de los/as adultos/as del equipo e incluyen sátiras sobre muchas de las crudas realidades cotidianas. Vale la coincidencia y resonancia con lo anteriormente desarrollado, respecto a lo que constituye un accionar quijotesco y la lucha contra las injusticias que, a la manera de un desplazamiento, toman a cargo los personajes ficcionales $\mathrm{Su}$ per-Villa y Puentechueka.

En el mismo hilván entran las actividades propuestas por el equipo donde se investigan y recrean historias de terror típicas del barrio (Lobizón, Llorona, Pombero, brujas, macumbas, fantasmas, demonios, etc.); y se cuentan otras, se personifican, escriben y publican en un "NotiPuente" con "misteriosos hechos en la Villa". Este bloque de actividades se produce contemporáneamente a la pregunta que debate el equipo sobre los relatos y la historicidad. ¿Cómo dar un salto desde los juegos más tradicionales con pelotas, hacia actividades que pongan en juego la capacidad/producción/elaboración discursiva de los/as niños/ as? Desde ya, participan de esa elaboración tanto niños/as como adultos/as. Se entrevé la forma re-creativa de ficcionar y horadar las "historias de terror" que llamamos encerronas trágicas; esas historias de terror de avasallante realidad que tienen como protagonistas a los/as mismos/as niños/as del Puente de Tierra.

\section{Equipo y producción de Salud Mental}

Como herramienta para la reflexión acerca de la relación entre los modos de trabajo descriptos y la producción de salud mental, la obra de Fernando Ulloa $(1995,2011)$ resulta fundamental. Él desarrolla una re-conceptualización de la Salud Mental desde la perspec- tiva psicoanalítica y un intento de situar una clínica a partir de síndrome de padecimiento, en escenario del campo sociocultural. La tensión dinámica de un sujeto al mismo tiempo social y singular, propicia el accionar del psicoanálisis en este campo de la numerosidad social que a su vez será recinto perelaborativo multiplicador.

\section{El término numerosidad hace referencia} a la sumatoria de sujetos que resulta cuando al dueto analista/analizante se le suma uno, más uno, más uno... (...) En el campo de la numerosidad social, cuentan tantos sujetos de cuerpo presente como sujetos hablantes cuentan. El primer cuentan alude a la mirada en reciprocidad, punto de partida de cualquier sujeto en tanto sujeto social. El segundo cuentan se refiere al discurso, ya que el discurso de un sujeto siempre tiene algo de singular; en ello radica, básicamente, el inicio de la singularidad de un sujeto, en su condición de pensante. (Ulloa, 2011, pp. 50-51)

El recurso de la perelaboración, entonces, constituye un recurso curativo propio de la condición humana y tiene un parentesco con el concepto de trabajo psíquico por el cual el aparato psíquico elabora los estímulos perturbadores. Esto cobra importancia en tanto el equipo de trabajo del Puente de Tierra desarrolla su práctica en contextos de pobreza y marginalidad, con una población y un equipo expuestos a un alto grado de crueldad.

Tanto el trabajo psicoanalítico como el proceder crítico pueden promover la perelaboración. En los modos de trabajo del equipo, están presentes estos dos recursos, ya que la práctica se despliega en la interdisciplina. Se instala un proceso que en primer término es eficaz sobre quienes sostienen la crítica $y$, 
luego, se enlaza al acontecer que apunta a los efectos a futuro de la práctica.

En la clínica para el abordaje de los espacios socioculturales que propone Ulloa (2011), se distingue una clínica que fluctúa entre la que se reconoce como de linaje médico, y otra propia del linaje psicoanalítico. En la vertiente política de la producción de Salud Mental, sitúa como operadores clínicos a todos/as aquellos/as que en este escenario actúan en los equipos asistenciales (evitando la injusta dicotomía que habla de profesiones universitarias y excluye a los niveles de enfermería, administración y mantenimiento).

Estos equipos debaten sus prácticas en común buscando producir una capacitación interdisciplinaria, pilar básico de una buena y sostenida Salud Mental, como producción cultural, para nada ajena a esa capacitación (Ulloa, 2011, p.75)

En función de lo desarrollado hasta aquí, en tanto el equipo de Puente de Tierra lleva adelante modos de trabajo colectivos, con instancias de debate y procederes críticos, poniendo en el centro de su tarea la actividad lúdica, recreativa y cultural; es posible inscribir su práctica cotidiana en el campo de producción de Salud Mental.

Además, se trata de una práctica comunitaria en salud/salud mental que se sostiene en una estrategia desde el pensar, en contraposición a una estrategia desde el saber (de la Aldea y Lewkowicz, 2004). Puente de Tierra despliega la potencia de construir en reciprocidad una comunidad de la que se es parte; haciendo con los/as otros/as y no por los/as otros/as; y dándose la posibilidad de pensar, de que la comunidad se piense, en términos de situación concreta. Este escenario rehúye a una lógica entre la omnipotencia y la impo- tencia, entre héroes y víctimas, como dos caras de la misma moneda que suprime al sujeto.

Se torna interesante la reflexión sobre las condiciones necesarias para sostener una práctica como Puente de Tierra. “¿Cuáles son las capacidades subjetivas que se requieren para habitar esos dispositivos? (...) el punto central es situar los obstáculos para que se constituya una subjetividad una comunidad de pensamiento capaz de habitar la situación existente." (p. 16) De esta manera, se trata de sostener los espacios de problematización y situar dispositivos para armar las condiciones prácticas que posibiliten que el pensamiento opere sobre el obstáculo, y no viceversa. El hecho de construir una subjetividad común, entonces, se escabulle respecto a dos de las posiciones dominantes; de idealización o de resignación.

De la subjetividad heroica impotente es posible deslizarse al gesto heroico, que lleva al hacer con otros y remite a lo maleable de la identidad propia, a la posibilidad de ser transformado por el hacer. Tal vez se pueda aventurar, además, que remite a la posibilidad de jugar, ficcionar, recrear, horadar, respecto a la realidad en contexto de adversidad (remitiendo a aquel primer vivir creador y los artificios desarrollados por el equipo Puente de Tierra). Con estos gestos se recupera la potencia de ir más allá de lo dado, en nombre de lo que puede haber, o desearíamos que haya.

\section{Reflexiones}

A partir de la caracterización del dispositivo y la puntualización de la dinámica cotidiana (centrada en el juego), es posible afirmar que los modos de trabajo del equipo toman las características mismas de la experiencia Puente de Tierra. Así, esos modos de trabajo, interdisciplinarios e interinstitucionales, se 
presentan con su cualidad de subjetivantes, $y$ con un marcado carácter situado (territorial). Además, se plasman como un determinado modo de construcción de vínculos en el que se hace hincapié en las relaciones de igualdad, dignidad, cooperación, confianza y respeto de las singularidades.

Los modos de trabajo del equipo analizados consisten, fundamentalmente, en las características de los vínculos entre los integrantes del equipo entre sí, con la población, y con el territorio; los acuerdos en torno al sostenimiento del espacio de juego, la formulación de sus objetivos y las modalidades que adquieren en la práctica; las actividades llevadas a cabo, como la materialización de los modos en que se da sentido a la práctica, tanto las instancias del equipo mismo (planificación, evaluación y supervisión) como las actividades en terreno y así también, los recursos y operaciones de los que se vale el equipo para llevarlas a cabo.

El equipo de Puente de Tierra despliega su trabajo cotidiano en una dinámica micropolítica en la cual el equipo mismo se constituye, singular y colectivamente, como productor y producido, en los modos de actuar y los procesos relacionales de los que forma parte. Este dispositivo lúdico es un espacio comprometido con la producción de sentidos para la práctica que lleva a cabo, operando en el campo de los procesos de subjetivación; los cuales no serían posibles sin una implicancia singular y colectiva por parte del equipo. Esta cuestión, lejos de constituirse en un mandato o una simple declaración de principios, se da en el marco de una unidad de trabajo de gestión colectiva, que apela a la dimensión productiva y generadora de la misma, produciendo búsqueda, movimiento y desestabilización de lo instituido. Así, instaura espacios (instancias) en los cuales se experimenta la toma de decisiones colectivas, se formulan proyectos, se procesa aquello que llega al equipo, $y$ se analiza la implicación con aquello que se produce. ¿Qué se procesa en las instancias? Lo externo, lo interno, las problemáticas territoriales, las propias implicaciones, lo político de la experiencia, lo subjetivo del equipo, el dolor ante la crueldad, las encerronas trágicas, etc. ¿Qué se produce en esas instancias? Puente de Tierra como proyecto, la revalorización de la palabra, el ejercicio reflexivo, las actividades para los/as chicos/as, los sentidos de la práctica, etc.

Respecto de la estrecha relación entre el proceso productivo y el producto; y teniendo en cuenta que este dispositivo trabaja con la producción de subjetivad -a la manera de una apuesta a la configuración de las tramas colectivas posibles para que el sujeto tome un lugar-, inevitablemente es un espacio donde los/as mismos/as trabajadores/ as toman su lugar como sujetos deseantes. Se instalan, además, los procesos necesarios para la producción de identidad, producción cultural, producción de saber interdisciplinario (saber curioso), producción de espacios públicos, etc.

En tiempos de profundo desamparo, Puente de Tierra se formula como una experiencia de construcción de amparos, reparos, a través del trabajo colectivo, la apuesta simbólica y la recreación de realidades, subjetividades y del espacio mismo. Afectado por las dificultades del trabajo en territorio, el equipo pone en juego el recurso de tercero de apelación, potenciando su capacidad crítica y autocrítica y rehuyendo de los efectos de la cultura de mortificación (Ulloa, 1995). De esta manera, los procesos de trabajo del equipo se llevan adelante junto a otros/as, que toman las características de sostén y resguardo.

Respecto a las vinculaciones con la producción de salud mental tal como se ha definido, la dimensión per-elaborativa, creativa y de pensamiento crítico es central tanto en los 
modos de trabajo del equipo como en la experiencia en sí. La práctica acontece desplegando sus efectos a futuro; y los miembros del equipo, como operadores clínicos, se disponen a una práctica de buen trato, construyendo escenarios de ternura. Este sentido per-sistente nos señala una intensidad sostenida en el tiempo, por parte de un equipo que esquiva la mortificación cultural y sostiene la "formidable inventiva de un colectivo en acción” (Fernández, en Ulloa, 2011, p.189).

Puente de Tierra ha representado una posibilidad de que la pregunta por las infancias y las juventudes permanezca abierta; como un puente hacia la pluralidad de sentidos, lo colectivo, las transformaciones, las singularidades y, centralmente, un puente hacia el juego.

El presente artículo intenta no redundar respecto a las adversidades que nos afectan y elegimos enfrentar en el trabajo con amplios sectores excluidos a la marginalidad y a la pobreza que deshumaniza; sino aportar, en cambio, algunos trazos para atravesarlas, a la manera de un puente. Lo esencial del proceder crítico del pensamiento: establecer las causas en el pasado que generan consecuencias en el futuro que cuestionan esas causas. (Ulloa, 1995) En este sentido, el proceso es inagotable y continuará, en adelante, atravesando otros puentes.

Ahora bien, interesa situar el hecho de que, si bien Puente de Tierra se ha desarrollado durante una década, en la actualidad ya no se lleve adelante. A menudo las tensiones con la política pública no pueden resolverse, la dificultad del trabajo en territorio impera y los conflictos específicos de cada institución imposibilitan la continuidad de espacios como éste (precarización del trabajo, rotación de personal, finalización de programas, demanda de atención o trabajo de oficina, desvalorización de prácticas en el barrio, etc.). Lo que deriva en la pregunta sobre la ineludible responsabilidad de las gestiones estatales y subraya el marco necesario, imprescindible, de políticas públicas que desplieguen recursos y lineamientos para el sostén de dispositivos como Puente de Tierra. Recuperar, construir y mantener el carácter público de un sector del territorio, como podría ser la canchita en el medio de la villa donde se despliega el juego de este equipo, debería estar en la agenda política para la construcción de ciudadanía. Muy por el contrario, experiencias o dispositivos territoriales, extramuros, suelen tejerse a contrapelo de la tendencia hegemónica de lo instituido a replegar la asistencia hacia dentro de los muros, a la preminencia de políticas represivas y a la renegación de los hechos, con su consecuente efecto siniestro.

Así las cosas, ¿Qué espacio queda para las infancias, para el juego, para el vivir creador, para la salud mental? ¿Qué lugar, para la potencialidad y el deber ético de trabajadores/as en el ámbito público?

Cabe también la pregunta sobre las situaciones donde el esfuerzo colectivo de un equipo no alcanza para sostener los dispositivos. ¿Qué hacer cuando al apelar a la dimensión tercera de lo estatal, las políticas públicas faltan o apuntalan su aspecto represivo? ¿Qué estrategias inventar para continuar el camino de producción de subjetividad y recuperación de lo público?

Este artículo se centra en una experiencia particular, pero similar a tantas otras que seguramente se dan a lo largo de diversos territorios. La importancia de registrarla y hacer circular sus modos se relaciona con valorar las instancias y estrategias desde el pensar de las que se sirve, y con transmitir la necesidad de organizar sistemáticamente el trabajo para defender dichas instancias de debate y decisión colectiva. El análisis realizado podría replicarse a otras experiencias territoriales, que cotidianamente son llevadas a cabo por traba- 
jadores/as en compromiso con los derechos humanos. Dispositivos como Puente de Tierra promueven la producción de salud mental en términos positivos, culturales y diversos.

Luego de profundizar el estudio sobre los modos de trabajo que permiten al equipo sostener esta experiencia territorial en contextos de dificultad interesa plantear el interrogante sobre las formas de institucionalizarlos. $\mathrm{Si}$ bien necesariamente, en una práctica situada, cada colectivo adopta su manera particular, resultaría interesante delinear una propuesta organizativa de modos de trabajo, para propiciar dichas producciones.

Durante la escritura de este artículo los diarios de la ciudad de Rosario informan sobre el asesinato a cuchilladas de un jovencito de veinte años, luego de una discusión con un vecino, en la villa en la que funcionó Puente de Tierra. Se trata de uno de los chicos que cuando niño participaba del dispositivo, el mismo protagonista de aquella escena que fue relatada aquí y quien eterniza ahora una historia singular signada por situaciones injustas y un trágico desenlace. Una vez más, el equipo es sujeto consciente de la crueldad con la que nuestra sociedad convive diariamente, confrontándose con un gran dolor. Una y otra vez, la experiencia común en Puente de Tierra los contacta entre sí a pesar de los tiempos de aislamiento $^{6}$; atravesando un dolor compartido como se atraviesa un puente que cruza un río, y al cruzarlo no lo anula y al mismo tiempo lo cruza. Ésa también es la función del conocimiento, opuesto a la tríada del conocimiento del cruel: que es la exclusión, el odio

\footnotetext{
${ }^{6}$ Transcurre el año 2020 signado por las profundas trasformaciones que conlleva la pandemia mundial por covid19. Aquí se refiere al aislamiento físico obligatorio. Es claro que en referencia al equipo persiste una capacidad, anclada en sus lazos, de sortear las distancias. La forma de contacto más asidua se vale de una aplicación de mensajería instantánea para teléfonos inteligentes.
}

y la eliminación. De alguna manera, también esta escritura intenta abonar a un puente para cruzar la crueldad; como lo hace Puente de Tierra para quienes lo habitaron.

A fin de cuentas, el estar afectados/as por las realidades adversas que viven grandes sectores de nuestra sociedad es un importante punto de resistencia y de potencia, con la posibilidad de hacer retroceder la intimidación y crear condiciones de ternura (Ulloa, 2003). Persistir en la apuesta al trabajo colectivo dentro, y fuera, de las instituciones públicas puede constituirse en el hilván que perdura y trasciende cada experiencia, y se convierte en una invitación a resistir, con toda la mar detrás, en contextos de tanto arrasamiento, intemperie y crueldad.

\section{Referencias}

- De la Aldea, E. y Lewkowicz, I. (2004). La subjetividad heroica. Un obstáculo en las prácticas comunitarias de la salud. Disponible en https://lacasona.org.ar/document/lasubjetividad-heroica/ 31/05/2019

- Equipo Puente de Tierra (2009) Proyecto Puente de tierra. Puente sobre el mundo. Documento de trabajo inédito.

- Franco, T. B. y Merhy, E. E. (2009) "Mapas analíticos: una mirada sobre la organización y sus procesos de trabajo." En Salud Colectiva, Vol. 5, Núm. 2. Pp. 181-194.

- Grande, S.; Mana, G. y Zampiero, G. (2018) "Lo grupal como marco: la tensión entre lo singular y lo colectivo." En Barquitos Pintados. Experiencia Rosario. Número 2. Pp.39-55.

- Jara H., O. (1994) Condiciones para poder sistematizar. En, Para sistematizar experiencias: una propuesta teórica y práctica. San José, CR: Alforja. Pp.74-85.

- (1994) ¿Cómo sistematizar? (una propues- 
ta en cinco tiempos). En, Para sistematizar experiencias: una propuesta teórica y práctica. San José, CR: Alforja. Pp.88-125.

- (2012) "Sistematización de experiencias, Investigación y Evaluación: aproximaciones desde tres ángulos." En, Revista Internacional sobre Investigación en Educación Global y para el Desarrollo, Número 1 - 2012. Pp. 56-70.

- Onocko Campos, R. (2002) La gestión: espacio de intervención, análisis y especificidades técnicas. Campinas. Disponible en https://logicasinstitucionalescpo.wordpress.com/2013/03/05/onoko-la-gestion/ 21/09/2020

- Real Academia Española (2019) Diccionario de la lengua española. Versión 23.3 en línea. Disponible en https://dle.rae.es 02/10/2020

- Ulloa, F. (1995). Novela Clínica psicoanaliti- ca. Historial de una práctica. Buenos Aires: Paidós.

- (2003) Escenarios de la ternura: resonancias en la educación maternal. Conferencia dictada en el Ciclo de Conferencias Proyecto Fortalecimiento de la Tarea Educativa en Instituciones Maternales. Buenos Aires, Argentina.

- (2011). Salud ele-Mental. Con toda la mar detrás. Buenos Aires: Libros del Zorzal.

- Winnicott, D. (1971) Realidad y Juego. Barcelona: Gedisa.

- Zelmanovich, P. (2003) Contra el desamparo. En I. Dussel \& S. Finocchio (Comp.), Enseñar Hoy. Una introducción a la Educación en tiempos de crisis. Buenos Aires: Fondo de Cultura Económica. Disponible en http://campuseducativo.santafe. gob.ar/wp-content/uploads/Contrael-desamparo.-Perla-Zelmanovich.pdf. $21 / 09 / 2020$ 